\title{
OSTEOMYELITIS OF THE PREMAXILLA*
}

BY

\author{
E. C. ALlibONE and C. P. MILlS \\ From the General Infirmary, Leeds
}

(RECEIVED FOR PUBLICATION FEBRUARY 21, 196́)

The superior maxilla forms the basis of the upper jaw. Osteomyelitis in this bone is a rare occurrence. Cavanagh (1960) found 152 cases in the literature but was able to add 24 of her own.

Most cases occur in the first three months of life. While the route of entry of the staphylococcus is disputed, the infection, once established, becomes widespread. Abscesses in relation to the floor of the orbit appear at the outer or inner canthi. Downward spread through the alveolus points in the region of the first deciduous molar tooth. Backward spread ruptures into the antrum and so drains into the nose. The clinical picture is that of an acutely ill feverish infant with a unilateral swelling of the face and eye, a swollen gum and a purulent nasal discharge all on the same side.

Osteomyelitis can, however, develop in relation to the upper incisor teeth, that is in the premaxillary portion of the superior maxilla. When this occurs the clinical picture is more localized and more benign. This observation is well known on the continent of Europe (Rickham, 1960). Grob (1957) mentions that in infants with complete hare-lip and cleft palate a much less virulent form of osteomyelitis is seen in the premaxilla.

\section{Case Reports}

Case 1. A twin, born in hospital, had thrived normally. In the second week of life he began to possett blood. By the end of the week the mother noticed a swelling of the upper gum on the right side. The same evening a tooth was noticed in the swelling as something white. Two days later the tooth came away, the swelling went down, but a discharging sinus persisted. While the gum was swollen the baby vomited frequently and had difficulty in sucking. Treatment was given at home by the general practitioner with oral penicillin.

At 3 weeks of age the baby was referred to the outpatient clinic. He appeared to be well. At the position of the right upper lateral incisor was a lentil sized granuloma. There was a little inspissated pus in the right

* A paper read at a meeting of the Paediatric Societies of the Birmingham, Leeds, Liverpool and Sheffield regions held in Leeds in October 1960. nostril. The airway appeared to be clear. When seen 10 days later a purulent nasal discharge had developed from which Staphylococcus aureus resistant to penicillin was cultured. After a further $\mathbf{1 0}$ days the baby sneezed out of the right nostril a sequestrum about the size of the erupted tooth (Fig. 1). After this episode local antiseptic treatment was stopped in favour of oral erythromycin which was given for three weeks. During this time the nasal discharge dried up. Recovery was uneventful. The remaining incisor teeth subsequently erupted normally.

Case 2. A triplet, whose mother was English and father Jamaican, was born in hospital, birth weight $5 \mathrm{lb} .1 \mathrm{oz}$. She did not gain weight quite so quickly as her two sibs and vomited about once a day for no discernible reason. At the age of 3 weeks the mother noticed spots of blood sometimes on the cheek and sometimes on the nightdress. The baby was admitted on the 25th day for general reasons. She continued to vomit readily and did not gain weight. At the age of 6 weeks she became feverish and the right side of the face swelled up. The next day pus was noticed to be oozing from the upper gum margin. Culture gave a growth of Staphylococcus aureus insensitive to penicillin. Erythromycin and novobiocin were given orally for eight weeks and then, after a three-week interval, for a further five weeks. The initial fever settled after a

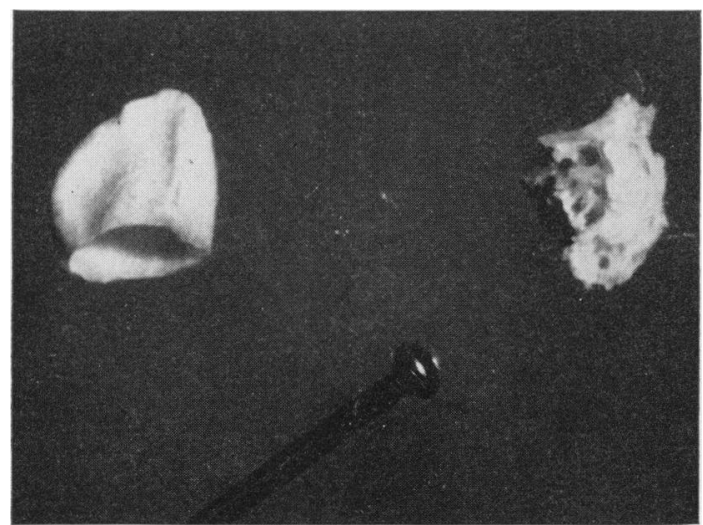

Fig. 1.-Case 1: The erupted tooth and the sequestrum which was subsequently extruded through the nostril. 


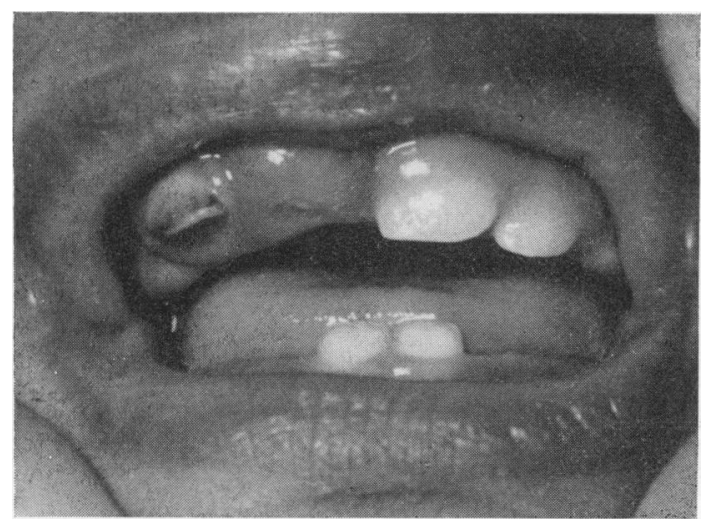

FIG. 2.-Case 2: The partially extruded and devitalized lateral incisor with delayed eruption of the central incisor of the same side. Condition at the age of 1 year.

few days. Two weeks later the right upper lateral incisor erupted and descended about half way. The baby thrived slowly. She still vomited readily.

At the age of 1 year the right upper lateral incisor was still in the same position. It was discoloured. Both left upper incisors had descended. The right upper central incisor had just cut the gum (Fig. 2).

Case 3. Paul H. was born in hospital. On arrival home on the 10th day the mother noticed an abscess on the upper gum which discharged pus from time to time. At the age of 3 weeks an 'abscess' appeared in the right nostril which was treated with oral penicillin by his family doctor. About this time the right eye became swollen and closed. Because of persistence of symptoms and some difficulty in breathing he was referred at the age of 5 weeks to a paediatric clinic. On examination he was pale but not toxic. There was a sanguinous discharge down the right nostril. The cheek and the right side of the nose were slightly swollen and there was a prominence the size of a pea near the inner canthus. The gums were not swollen, but the right upper central incisor was just visible (Fig. 3).

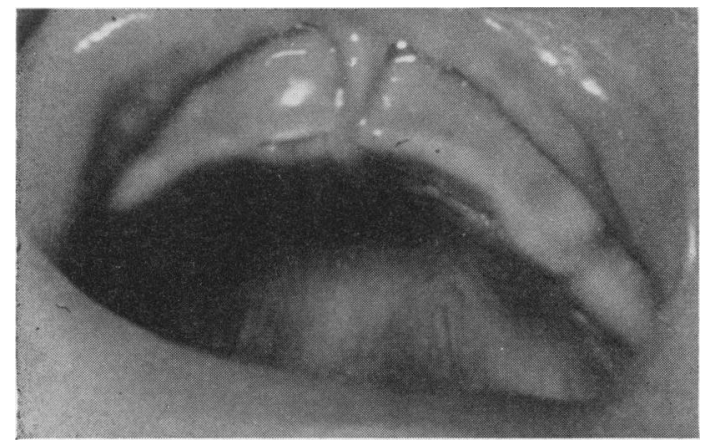

FIG. 3.-Case 3: Premature eruption of the right upper central incisor at the age of 5 weeks.
He was admitted to hospital. Staphylococcus aureus resistant to penicillin was cultured from nose and throat. He was treated with erythromycin and novobiocin for eight weeks. Because the swelling of the face and the nasal discharge persisted he was examined under anaesthesia. A sinus was found in the floor of the nose. The partially erupted incisor was lying in a bed of pus. It was extracted to facilitate drainage. He was then discharged.

He remained well. The haemorrhagic discharge from the nose persisted intermittently. When it stopped the swelling of the inner canthus became more noticeable and discharged. By the age of 5 months the right upper lateral incisor had erupted, but it came down crooked.

\section{Discussion}

The three cases described are similar in their presentation in early infancy as a swelling of the alveolus over one of the upper incisor teeth with subsequent eruption of the relevant tooth. In the first two cases the infection remained limited to the premaxilla. In the third case there was evidence of spread after the lapse of a week. The organism was in each case Staphylococcus aureus, coagulase positive and resistant to penicillin. The systemic reaction was slight.

The involvement of the premaxilla does not support the theory that osteomyelitis of the maxilla starts as an infection of the antrum. That in each case only one tooth bud was completely devitalized favours the concept that the infection settles in a tooth bud (Wilensky, 1932; Asherson, 1939; McCash and Rowe, 1953; Gregson, 1958).

The occurrence of hare-lip and cleft palate indicates a failure of fusion in the embryo of the maxillary and fronto-nasal processes. At birth there is normally no evidence of cleavage. Nevertheless, the tendency for infection to remain localized to the premaxilla in the above cases suggests the existence of a vascular watershed which forms a functional barrier to the spread of infection.

\section{Summary}

Three cases of osteomyelitis arising in the premaxilla are described. The clinical picture is contrasted with infection starting in the main portion of the maxilla.

We are greatly indebted to Mr. S. R. Fell, F.D.S.R.C.S., and to Mr. D. Jackson, D.D.S., for their help and advice.

\section{REFERENCES}

Asherson, N. (1939). Acute osteomyelitis of the superior maxilla in young infants. J. Laryng., 54, 691. 
Cavanagh, F. (1960). Osteomyelitis of the superior maxilla in infants. A report on 24 personally treated cases. Brit. med.J., $1,468$.

Gregson, A. E. W. (1958). Acute osteomyelitis of the maxilla in infants. $J$. Laryng., 72, 312.

Grob, M. (1957). Lehrbuch der Kinderchirurgie, p. 114. Thieme, Stuttgart.
McCash, C. R. and Rowe, N. L. (1953). Acute osteomyelitis of the maxilla in infancy. J. Bone Jt Surg., 35B, 22.

Rickham, P. P. (1960). Personal communication.

Wilensky, A. O. (1932). The pathogenesis and treatment of acute osteomyelitis of the jaws in nurslings and in infants. Amer. $J$. Dis. Child., 43, 431 . 